\title{
Quantum Photonic Interface for Tin-Vacancy Centers in Diamond
}

\author{
Alison E. Rugar $\odot,{ }^{1, *}$ Shahriar Aghaeimeibodi® ${ }^{1, *}$ Daniel Riedel $\odot,{ }^{1, *}$ Constantin Dory $\odot,{ }^{1}$ Haiyu Lu๑, ${ }^{2,3,4}$ \\ Patrick J. McQuade, ${ }^{4,5}$ Zhi-Xun Shen ${ }^{2,3,4,6}$ Nicholas A. Melosh ${ }^{4,5}$ and Jelena Vučković ${ }^{1, \dagger}$ \\ ${ }^{1}$ E. L. Ginzton Laboratory, Stanford University, Stanford, California 94305, USA \\ ${ }^{2}$ Department of Physics, Stanford University, Stanford, California 94305, USA \\ ${ }^{3}$ Geballe Laboratory for Advanced Materials, Stanford University, Stanford, California 94305, USA \\ ${ }^{4}$ Stanford Institute for Materials and Energy Sciences, SLAC National Accelerator Laboratory, \\ Menlo Park, California 94025, USA \\ ${ }^{5}$ Department of Materials Science and Engineering, Stanford University, Stanford, California 94305, USA \\ ${ }^{6}$ Department of Applied Physics, Stanford University, Stanford, California 94305, USA
}

(Received 11 March 2021; accepted 26 May 2021; published 26 July 2021)

\begin{abstract}
The realization of quantum networks critically depends on establishing efficient, coherent light-matter interfaces. Optically active spins in diamond have emerged as promising quantum nodes based on their spin-selective optical transitions, long-lived spin ground states, and potential for integration with nanophotonics. Tin-vacancy $\left(\mathrm{SnV}^{-}\right)$centers in diamond are of particular interest because they exhibit narrow-linewidth emission in nanostructures and possess long spin coherence times at temperatures above $1 \mathrm{~K}$. However, a nanophotonic interface for $\mathrm{SnV}^{-}$centers has not yet been realized. Here, we report cavity enhancement of the emission of $\mathrm{SnV}^{-}$centers in diamond. We integrate $\mathrm{SnV}^{-}$centers into onedimensional photonic crystal resonators and observe a 40-fold increase in emission intensity. The Purcell factor of the coupled system is 25 , resulting in a channeling of the majority of photons (90\%) into the cavity mode. Our results pave the way for the creation of efficient, scalable spin-photon interfaces based on $\mathrm{SnV}^{-}$centers in diamond.
\end{abstract}

DOI: 10.1103/PhysRevX.11.031021

Subject Areas: Optics, Photonics, Quantum Information

\section{INTRODUCTION}

The basis of scalable quantum networks is nodes with optically accessible, long-lived quantum memories coupled to efficient photonic interfaces [1]. A critical milestone toward the implementation of a quantum network is the development of an efficient, coherent light-matter interface $[2,3]$. Such an interface can be implemented by coupling optically active spin qubits to nanophotonic cavities. By strongly confining optical fields, nanocavities enhance the coherent emission of embedded qubits and channel the emitted photons into a single optical mode. Diamond hosts a number of color centers that are excellent optically interfaced spin qubit candidates [4-6]. The most established of these color centers is the nitrogen-vacancy $\left(\mathrm{NV}^{-}\right)$ center, which has enabled seminal experiments such as heralded long-distance entanglement [7] and entanglement

\footnotetext{
These authors contributed equally to this work. †jela@stanford.edu
}

Published by the American Physical Society under the terms of the Creative Commons Attribution 4.0 International license. Further distribution of this work must maintain attribution to the author(s) and the published article's title, journal citation, and DOI. distillation [8]. Unfortunately, fabrication-induced charge noise degrades the optical coherence of $\mathrm{NV}^{-}$centers and thus precludes the integration of $\mathrm{NV}^{-}$centers with nanophotonic cavities $[9,10]$. The entanglement rates in these experiments are therefore limited by the small fraction of coherent emission into the $\mathrm{NV}^{-}$zero-phonon line (ZPL).

Group-IV color centers in diamond are significantly less sensitive to local charge fluctuations due to their inversionsymmetric structure [11]. As a consequence, these color centers exhibit nearly lifetime-limited linewidths in nanostructures despite the use of invasive plasma etching techniques [12-14]. While silicon-vacancy $\left(\mathrm{SiV}^{-}\right)$centers coupled to nanophotonic resonators have enabled the demonstration of high-fidelity single-shot readout and nuclear spin memory-enhanced quantum communication [15], $\mathrm{SiV}^{-}$centers suffer from low quantum efficiency, and their excellent spin properties are accessible only under high strain [16] or at millikelvin temperatures [17]. Fortunately, other group-IV color centers in diamond are expected to share many of the favorable properties of $\mathrm{SiV}^{-}$ centers but at higher temperatures [18]. Tin-vacancy $\left(\mathrm{SnV}^{-}\right)$centers $[13,19-21]$ stand out because of their high quantum efficiency [19] and long spin coherence times at cryogenic temperatures above $1 \mathrm{~K}$ [13]. Despite their promise as optically active spin qubit candidates, the 


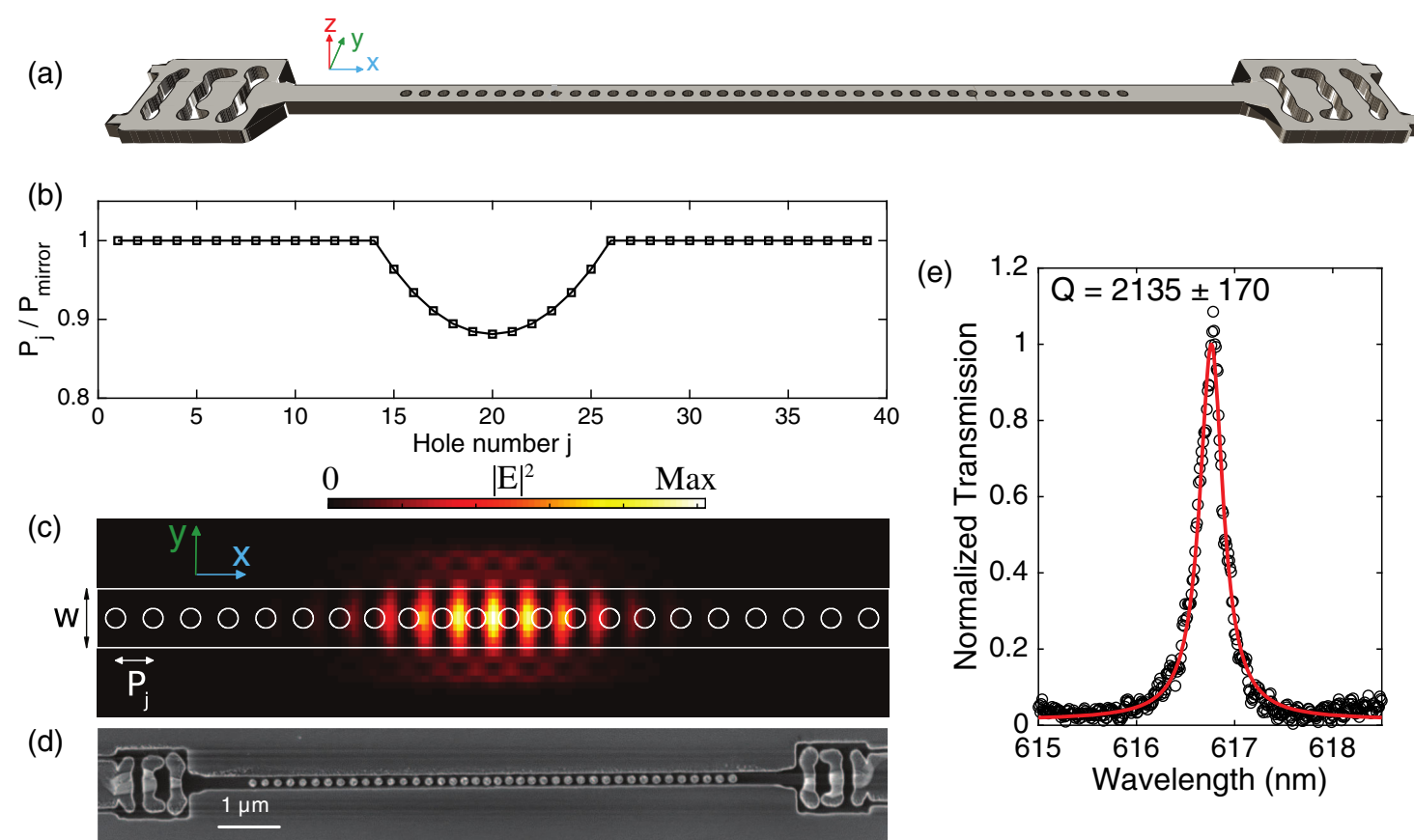

FIG. 1. Design and simulation of the nanobeam photonic crystal cavity. (a) Schematic of the proposed one-dimensional photonic crystal cavity. Two inverse-designed vertical couplers on either end of the beam enable the coupling of light into and out of the device. (b) Spacing between the holes for different hole numbers. A quadratic taper over the center 12 holes creates the cavity. (c) Finitedifference time-domain simulation of electric field magnitude squared $|E|^{2}$ showing confinement of light in the center of the cavity. (d) Scanning electron microscope image of a fabricated device. (e) Spectrum of a cavity mode of a representative device obtained from a transmission measurement through the device. The quality factor of this device is $Q=2135 \pm 170$.

critical step of incorporating $\mathrm{SnV}^{-}$centers into cavities has yet to be realized.

In this article, we report the coupling of the ZPL emission of $\mathrm{SnV}^{-}$centers to one-dimensional photonic crystal cavities. We fabricate a large array of devices on a single chip using a fabrication technique based on the quasi-isotropic diamond undercut method [22-26]. By tuning the cavity into resonance with the color center, we demonstrate a 40-fold increase in $\mathrm{SnV}^{-}$center emission intensity. Furthermore, we observe a tenfold reduction in the $\mathrm{SnV}^{-}$center excited-state lifetime, corresponding to a Purcell factor of 25 . Because of this enhancement, the majority of photons are emitted into the cavity mode via the ZPL $(\beta=90 \%)$. With their excellent optical properties [19] and competitive spin coherence times accessible without a dilution refrigerator [13], $\mathrm{SnV}^{-}$centers integrated with nanocavities constitute a promising platform for quantum networks.

\section{RESULTS}

Our cavities are based on one-dimensional photonic crystals where a periodic array of circular holes etched into a suspended diamond waveguide creates a photonic band gap [27]. As illustrated in Fig. 1(a), we implement inverse-designed vertical couplers on either end of the cavity to facilitate the in- and out-coupling of the light [26].
Figure 1(b) shows the designed spacing between the holes for a device with 20 holes on each side. We quadratically taper the spacing between the 12 central holes from $P_{\text {mirror }}$ in the mirror section to a reduced period $P_{\text {center }}=0.88 P_{\text {mirror }}$ in the center of the cavity to confine the light. We select the waveguide width $w=300 \mathrm{~nm}$, thickness $h=200 \mathrm{~nm}$, period $P_{\text {mirror }}=190 \mathrm{~nm}$, and hole radius $r=57 \mathrm{~nm}$ in order to have a resonance around $620 \mathrm{~nm}$ of high quality factor $(Q)$ and small mode volume $\left(V_{\text {mode }}\right)$. We simulate the performance of our design using the three-dimensional finitedifference time-domain method (Lumerical). Figure 1(c) illustrates the simulated $|E|^{2}$ for the above parameters with a simulated $Q=2 \times 10^{5}$ and $V_{\text {mode }}=0.56(\lambda / n)^{3}$.

Next, we fabricate our photonic crystal cavities. We first generate $\mathrm{SnV}^{-}$centers at a depth of $90 \mathrm{~nm}$ with our recently developed shallow ion implantation and growth method [28]. We then perform a fabrication routine based on quasiisotropic etching [22-26] to create a large matrix of devices. In order to account for shifts of the cavity resonance wavelength caused by fabrication imperfections, we vary $P_{\text {mirror }}$ by $\pm 20 \%$. We also vary the number of mirror periods. Figure 1(d) displays a scanning electron microscope (SEM) image of a representative fabricated cavity. Further details on color center generation and device fabrication are provided in the Appendix A.

We characterize our devices at cryogenic temperatures (Montana Instruments, $\sim 5 \mathrm{~K}$ ) in a home-built confocal 

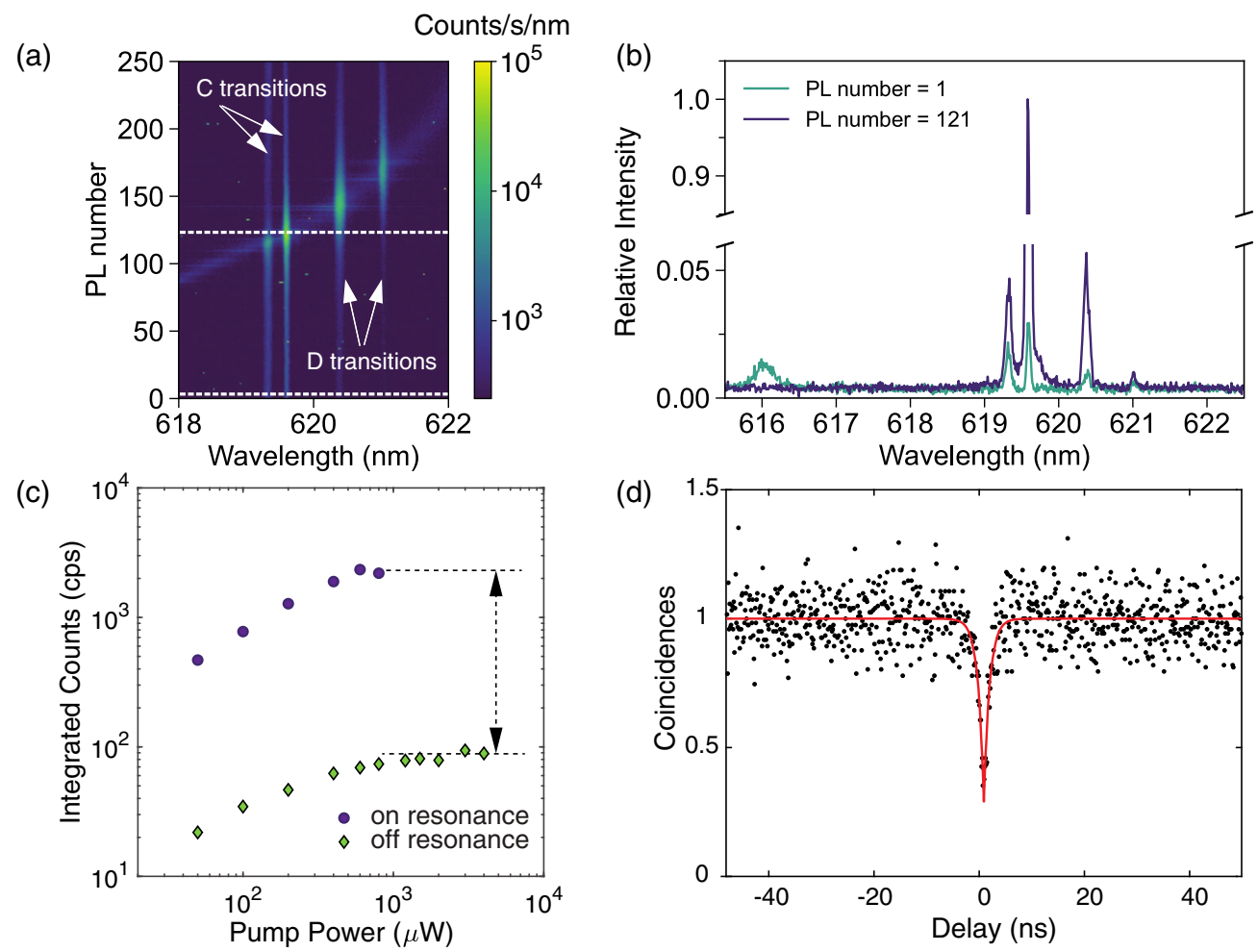

FIG. 2. Intensity enhancement of $\mathrm{SnV}^{-}$centers. (a) Consecutive PL spectra acquired while gas tuning the cavity through resonance with the ZPLs of the two $\mathrm{SnV}^{-}$centers. Two horizontal white dashed lines indicate locations of line cuts examined in (b). (b) Two line cuts acquired when the cavity mode is $3.6 \mathrm{~nm}$ blue detuned from the $C$ transition (teal) and when the cavity mode is resonant with the $C$ transition of interest at $619.6 \mathrm{~nm}$ (purple). (c) Saturation of the $\mathrm{SnV}^{-}$center when cavity mode is $2.72 \mathrm{~nm}$ blue detuned from the resonance (green diamonds) and when the cavity mode is resonant with the $C$ transition at $619.6 \mathrm{~nm}$ (purple circles). In the resonant case the maximum achieved intensity of the $C$ transition of the $\mathrm{SnV}^{-}$center is about 30 times greater than when the cavity is off resonance. (d) Second-order autocorrelation measurement of $\mathrm{SnV}^{-}$center when the cavity mode is resonant with the $C$ transition. A fit (red) to the data (black) reveals a $g^{(2)}(0)=0.29 \pm 0.08$.

microscope setup. We look for devices that have a cavity mode that is slightly blueshifted with respect to the optical transitions of the $\mathrm{SnV}^{-}$center so that we can eventually use gas tuning to tune the cavity mode into resonance with the $\mathrm{SnV}^{-}$center ZPLs (see Appendix B). We also check the devices for $\mathrm{SnV}^{-}$center photoluminescence (PL) signal.

To determine the quality factor and resonance wavelength of our cavities, we couple a supercontinuum laser through the vertical couplers and perform a broadband transmission measurement. Figure 1(e) shows the measured transmission spectrum of a suitable device $\left(P_{\text {mirror }}=228 \mathrm{~nm}\right.$ and 14 holes $)$, featuring a quality factor of $2135 \pm 170$ at $616.76 \mathrm{~nm}$.

Next, we perform PL spectroscopy (see Appendix B) on the device to verify the presence of $\mathrm{SnV}^{-}$centers in the device. Figure 2(a) displays a heat map of consecutive PL spectra. The four vertical lines apparent in Fig. 2(a) are the ZPLs of two $\mathrm{SnV}^{-}$centers present in the cavity. At $5 \mathrm{~K}$, individual $\mathrm{SnV}^{-}$centers have two prominent ZPLs centered about $620 \mathrm{~nm}$, known as the $C$ and $D$ transitions [19], where $C$ transitions are higher in energy than $D$ transitions. For the remainder of this article, we focus on the $C$ transition located at $619.6 \mathrm{~nm}$, which has the highest count rate.

We characterize the intensity of the $C$ transition at different detunings between the cavity mode and the $C$ transition. In Fig. 2(a), the cavity mode starts blue detuned from the $\mathrm{SnV}^{-}$center ZPLs. The cavity mode is then tuned through the ZPLs of the $\mathrm{SnV}^{-}$centers by argon gas condensation. We examine in Fig. 2(b) two spectra from Fig. 2(a) corresponding to the white dashed lines: one spectrum in which the cavity is $3.6 \mathrm{~nm}$ blue detuned from the $C$ transition of interest (PL number $=1$ ) and another in which the cavity mode is resonant with the $C$ transition at $619.6 \mathrm{~nm}$ (PL number = 121). Between the off- and onresonance cases shown in Fig. 2(b), the intensity of the $C$ transition increases by a factor of $40 \pm 4$, indicating that this $\mathrm{SnV}^{-}$-cavity system has a large Purcell factor. The intensity enhancements of the other $C$ transition at $619.3 \mathrm{~nm}$ and the $D$ transitions at 620.4 and $621.0 \mathrm{~nm}$ are, respectively, $11 \pm 2,56 \pm 11$, and $42 \pm 17$.

To further examine the intensity enhancement, we characterize the emission intensity of the $C$ transition as a function of excitation power when the cavity mode is off 

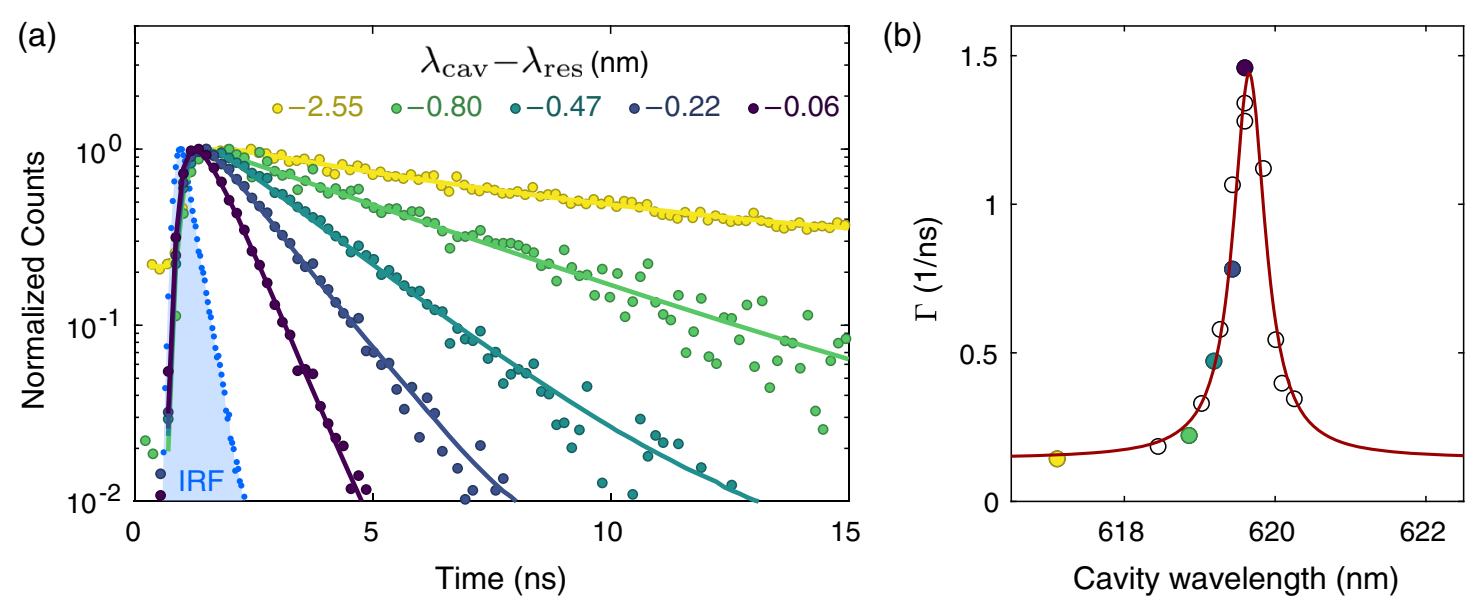

FIG. 3. Time-resolved PL measurements. (a) Representative lifetime measurements at different cavity wavelengths. To fit the data we convolve a single-exponential decay with the instrument response function (IRF) of our detectors (blue). (b) PL recombination rates of the studied $\mathrm{SnV}^{-}$center plotted as a function of cavity resonance wavelength $\left[\Gamma\left(\lambda_{\text {cav }}\right)\right]$. The data (circles) are fit with a Lorentzian model (red curve) to quantify the enhancement provided by the cavity. Filled circles correspond to lifetime data of the same color shown in (a).

and on resonance with the $C$ transition. The resulting saturation data are presented in Fig. 2(c). We observe up to 30 times greater intensity for the resonant case than for the off-resonance case. At high excitation powers, the deposited Ar starts to evaporate and the cavity becomes detuned from the $C$ transition. This effect limits the range of excitation powers that we can use without removing $\mathrm{Ar}$ to $\sim 1 \mathrm{~mW}$. This large intensity contrast combined with the 40-fold intensity enhancement observed in Fig. 2(b) indicates that the $\mathrm{SnV}^{-}$-cavity system under study has a large Purcell factor, which we will later quantify.

We confirm that the enhanced $\mathrm{SnV}^{-}$center is a singlephoton source by performing a Hanbury Brown and Twiss experiment with the cavity mode resonant with the $C$ transition. The resulting second-order autocorrelation data, shown in Fig. 2(d), displays a distinct antibunching dip. A fit to the data with a function of the form $g^{(2)}(\tau)=$ $1-\left[1-g^{(2)}(0)\right] e^{-|\tau| / \tau_{0}}$, where $\tau$ is the delay between detection events, reveals $\tau_{0}=(1.09 \pm 0.2) \mathrm{ns}$ and $g^{(2)}(0)=$ $0.29 \pm 0.08$, indicating that the enhanced $C$ transition is emission from a single quantum emitter. We attribute the nonideal $g^{(2)}(0)$ value to limited time resolution and, primarily, dark counts in our detectors. To examine the effect of time resolution on our $g^{(2)}(0)$ value, we fit to the data a convolution of the function $g^{(2)}(\tau)$ with a Gaussian function. We fix the Gaussian full width at half maximum to $0.40 \mathrm{~ns}$, which was extracted from a Gaussian fit to the instrument response function (IRF) measured below. Using $g_{\text {decon }}^{(2)}(0)$ and $\tau_{0 \text {,decon }}$ as fitting parameters, we find the deconvolved values $g_{\text {decon }}^{(2)}(0)=$ $0.26 \pm 0.10$ and $\tau_{0, \text { decon }}=(0.98 \pm 0.19) \mathrm{ns}$.

To quantify the Purcell enhancement of the system, we measure the excited-state lifetime of the $\mathrm{SnV}^{-}$center for different cavity resonance wavelengths $\left(\lambda_{\text {cav }}\right)$ through time-resolved PL measurements (see Appendix B). To tune the cavity resonance wavelength, we inject Ar gas and determine $\lambda_{\text {cav }}$ using a reflectivity measurement prior to each lifetime measurement. Figure 3(a) displays representative lifetime measurements at different cavity wavelengths integrated for $100 \mathrm{~s}$. To remove background contributions we subtract an off-resonant measurement $\left(\lambda_{\text {cav }}=616.1 \mathrm{~nm}\right)$ acquired under the same experimental conditions. We fit the data to a single exponential decay convolved with the IRF of our detectors. We then extract the excited-state decay rate $\Gamma\left(\lambda_{\text {cav }}\right)$ from the respective lifetime fits. A comprehensive study of the effect of the cavity on the $\mathrm{PL}$ decay rates of the $\mathrm{SnV}^{-}$center is shown in Fig. 3(b). To get a better estimate for the nonresonant radiative decay rate $\Gamma_{\text {off }}$, we perform an additional measurement at $\lambda_{\text {cav }}=$ $617.1 \mathrm{~nm}$ with an increased integration time (1000 s) and use a single-mode fiber for detecting the PL. We find $\tau_{617.1 \mathrm{~nm}}=(6.980 \pm 0.078) \mathrm{ns}$, which is comparable to previously reported lifetimes for $\mathrm{SnV}^{-}$centers close to the diamond bulk surface [21]. When the cavity mode is resonant with the $C$ transition, the lifetime is strongly reduced to $\tau_{619.6 \mathrm{~nm}}=(0.685 \pm 0.014) \mathrm{ns}$.

We determine the relevant system parameters by fitting the data in Fig. 3(b) to a Lorentzian model $\Gamma\left(\lambda_{\text {cav }}\right)=\Gamma_{\text {off }}+$ $\Gamma_{\mathrm{cav}} \delta \lambda_{\text {cav }}^{2} /\left[4\left(\lambda_{\mathrm{cav}}-\lambda_{\text {res }}\right)^{2}+\delta \lambda_{\text {cav }}^{2}\right]$ [29]. Here, the off-resonance decay rate is fixed to $\Gamma_{\text {off }}=\Gamma_{617.1 \mathrm{~nm}}=0.143 \mathrm{~ns}^{-1}$. We find a cavity-induced decay rate of $\Gamma_{\text {cav }}=(1.30 \pm$ $0.16) \mathrm{ns}^{-1}$ corresponding to an on-resonance decay rate of $\Gamma_{\text {on }}=\Gamma_{\text {off }}+\Gamma_{\text {cav }}=(1.44 \pm 0.16) \mathrm{ns}^{-1}$. The width and center wavelength of the Lorentzian fit are $\delta \lambda_{\text {cav }}=(0.260 \pm$ $0.040) \mathrm{nm}$ and $\lambda_{\text {res }}=(619.656 \pm 0.032) \mathrm{nm}$, respectively.

On resonance, the overall lifetime reduction caused by the interaction of the $\mathrm{SnV}^{-}$center with the cavity is given 
by $\tau_{\text {off }} / \tau_{\text {on }}=\Gamma_{\text {on }} / \Gamma_{\text {off }}=10.1 \pm 1.2$. From the measured cavity-induced decay rate $\Gamma_{\text {cav }}=F_{P}^{c} \xi_{c} \Gamma_{\text {off }}$, we infer the Purcell factor of the $C$ transition to be $F_{P}^{c}=24.8 \pm 3.0$. Here, $\xi_{c}=0.36$ is a factor that corrects for the nonunity probability of the $\mathrm{SnV}^{-}$center relaxing radiatively via the $C$ transition. $\xi_{c}$ is a product of quantum efficiency (80\% [19]), Debye-Waller factor (57\% [21]), and branching ratio into the $C$ transition (80\%, extracted from an off-resonance PL spectrum of this emitter). The figure of merit of our system $\beta$, the resulting probability of an excited-state decaying through emission into the cavity mode via the $C$ transition, is approaching unity: $\beta=$ $\Gamma_{\text {cav }} / \Gamma_{\text {on }}=(90.1 \pm 1.1) \%$.

To benchmark our system performance we calculate the theoretical Purcell enhancement for an emitter positioned at the maximum cavity field. The Lorentzian fit to $\Gamma\left(\lambda_{\text {cav }}\right)$ yields a cavity $Q$ factor of $2384 \pm 366$ [Fig. 3(b)] which is consistent with the transmission measurement in Fig. 1(e). The theoretical Purcell factor of our device is thus $F_{P}^{\text {theor }}=3 /\left(4 \pi^{2}\right) Q / V_{\text {mode }}(\lambda / n)^{3}=302 \pm 46$. Accounting for the angular mismatch between cavity polarization of $\langle 110\rangle$ and $\mathrm{SnV}^{-}$center symmetry axis along $\langle 111\rangle$ $\left(\cos ^{2} \phi=2 / 3\right)$, we infer that the studied $\mathrm{SnV}^{-}$experiences only $(12.3 \pm 2.4) \%$ of the maximum cavity field $\left|E_{\max }\right|^{2}$. This discrepancy hints at a displacement of the studied emitter from the field maximum.

\section{CONCLUSION}

We have demonstrated the Purcell enhancement of a $\mathrm{SnV}^{-}$center in a diamond photonic crystal cavity. With our $\mathrm{SnV}^{-}$-cavity system, we can achieve a 40 -fold increase in emission intensity into the $C$ transition. The system displays a lifetime reduction of 10 and a Purcell factor of 25 . As a result, $90 \%$ of the PL emission is channeled through the ZPL into the cavity mode, enabling a ZPL photon creation rate in excess of $1 \mathrm{GHz}$. The Purcell factor could be further increased by improving the $Q / V_{\text {mode }}$ ratio of the cavities $[23,25]$. On the chip fabricated in this work, there were other cavities with higher $Q$ factors $(Q \sim 5000)$ than the cavity studied in this paper. Additionally, deterministic positioning of the emitter can improve the yield of devices with large Purcell enhancement. Sub-10-nm placement accuracy can be achieved by combining our shallow ion implantation and growth technique [28] with implantation masks [30-32]. Cooperativities in excess of 100 have been achieved with $\mathrm{SiV}^{-}$centers by combining state-of-the-art $Q$ factors with deterministic emitter placement [15]. For their use in extended quantum networks, $\mathrm{SnV}^{-}$center $\mathrm{ZPL}$ photons need to be efficiently coupled into an optical fiber network [33]. To that end, the main loss channel of the coupled system needs to be transmission into the waveguide mode. Waveguide-to-fiber coupling efficiencies of $>90 \%$ can be achieved using fiber tapers [34]. As an alternative, optimized inverse-designed vertical couplers have the advantage of featuring a small footprint while potentially exhibiting similar coupling efficiencies $>85 \%$ [26]. Combining our $\mathrm{SnV}^{-}$-cavity systems with on-chip photonic architectures via hybrid integration techniques would enable large-scale quantum information processing systems $[12,35,36]$. Our work paves the way toward establishing a coherent and efficient spin-photon interface based on diamond color centers without the need for dilution refrigerators.

\section{ACKNOWLEDGMENTS}

This work is financially supported by U.S. Army Research Office (ARO) (Grant No. W911NF-13-10309); National Science Foundation (NSF) RAISE TAQS (Grant No. 1838976); DOE BES (Award No. DESC0020115); U.S. Air Force Office of Scientific Research (AFOSR) DURIP (Grant No. FA9550-16-1-0223). Stanford Institute for Materials and Energy Sciences (SIMES) research is supported by the Division of Materials Science and Engineering, Office of Basic Energy Sciences, U.S. DOE, and SLAC LDRD. A. E. R. acknowledges support from the National Defense Science and Engineering Graduate (NDSEG) Fellowship Program, sponsored by the U.S. Air Force Research Laboratory (AFRL), the Office of Naval Research (ONR), and the U.S. Army Research Office (ARO). S. A. acknowledges support from the Bloch postdoctoral fellowship in quantum science and engineering from Stanford Quantum Fundamentals, Architecture and Machines initiative (Q-FARM). D. R. acknowledges support from the Swiss National Science Foundation (Project No. P400P2_194424). C. D. acknowledges support from the Andreas Bechtolsheim Stanford Graduate Fellowship and the Microsoft Research Ph.D. Fellowship. Part of this work was performed at the Stanford Nanofabrication Facility (SNF) and the Stanford Nano Shared Facilities (SNSF), supported by the NSF under Grant No. ECCS-2026822.

Note added.- Recently, we became aware of a similar, very recent work [37].

\section{APPENDIX A: DEVICE FABRICATION}

We fabricate our nanophotonic resonators from an electronic-grade single-crystal diamond plate (Element Six). The chip is first cleaned in a boiling tri-acid solution (1:1:1 sulfuric/nitric/perchloric acids). We then remove the top $500 \mathrm{~nm}$ of the chip with an oxygen $\left(\mathrm{O}_{2}\right)$ plasma etch. By employing our recently developed shallow ion implantation and growth (SIIG) method [28] we create a $\delta$-doped layer of high-quality $\mathrm{SnV}^{-}$centers. Here, ${ }^{120} \mathrm{Sn}^{+}$ ions are implanted shallowly using low implantation energy $(1 \mathrm{keV})$ with a dose of $5 \times 10^{11} \mathrm{~cm}^{-2}$. Ion implantation was performed by CuttingEdge Ions. A thin film $(90 \mathrm{~nm})$ of high-quality diamond material is subsequently grown by microwave-plasma chemical vapor deposition (Seki 
Diamond Systems SDS 5010; $300 \mathrm{sccm} \mathrm{H}_{2}, 0.5 \mathrm{sccm} \mathrm{CH}_{4}$, stage temperature of $650{ }^{\circ} \mathrm{C}$, microwave power of $1100 \mathrm{~W}$, and pressure of 23 Torr).

We fabricate our photonic devices via the quasi-isotropic etching technique [22-26]. First, $200 \mathrm{~nm}$ of $\mathrm{Si}_{x} \mathrm{~N}_{y}$ are grown via plasma-enhanced chemical vapor deposition. The structures are then patterned in hydrogen silsesquioxane FOx-16 via electron-beam lithography. The $\mathrm{Si}_{x} \mathrm{~N}_{y}$ is then etched with $\mathrm{SF}_{6}, \mathrm{CH}_{4}$, and $\mathrm{N}_{2}$ reactive ion etch (RIE). We use the patterned $\mathrm{Si}_{x} \mathrm{~N}_{y}$ layer as an etch mask for the diamond substrate. The diamond is etched with an anisotropic $\mathrm{O}_{2}$ RIE. We then grow $30 \mathrm{~nm}$ of $\mathrm{Al}_{2} \mathrm{O}_{3}$ via atomic layer deposition. The horizontal planes of the $\mathrm{Al}_{2} \mathrm{O}_{3}$ layer are removed with a $\mathrm{Cl}_{2}, \mathrm{BCl}_{2}$, and $\mathrm{N}_{2} \mathrm{RIE}$ so that only the sidewalls of the diamond structures are covered by $\mathrm{Al}_{2} \mathrm{O}_{3}$. Using a second anisotropic $\mathrm{O}_{2}$ RIE we expose bare diamond sidewalls. The quasi-isotropic etch [22-26] step is now performed to undercut the structures. This $\mathrm{O}_{2}$ plasma etch step is performed at high temperature $\left(300{ }^{\circ} \mathrm{C}\right)$ with zero forward bias and high inductively coupled plasma power [22-26]. This etch progresses preferentially along the $\{110\}$ planes [23]. Once the nanobeam waveguides have been released and etched to the desired thickness, as validated by high-voltage SEM, the sample is soaked in hydrofluoric acid to remove the $\mathrm{Si}_{x} \mathrm{~N}_{y}$ and $\mathrm{Al}_{2} \mathrm{O}_{3}$ etch masks.

\section{APPENDIX B: MEASUREMENTS SETUPS}

All measurements were conducted in a home-built confocal microscope setup, with the sample cooled to $\sim 5 \mathrm{~K}$ in a Montana Instruments Cryostation.

To tune the cavity mode, we employ gas tuning. Argon gas is injected into the cryostat and freezes onto the devices. Condensed Ar increases the effective refractive index of the device and thus redshifts the cavity resonance wavelength. Gas deposition can be reversed by heating, either locally with a laser or chipwide with a heated stage.

For all PL measurements presented in Fig. 2, a continuous-wave, 532-nm laser filtered by a $532 \pm 3-\mathrm{nm}$ bandpass filter is used to excite the $\mathrm{SnV}^{-}$centers. For the PL data presented in Fig. 2(a), the excitation power was $500 \mu \mathrm{W}$. Before being coupled into a single-mode fiber, the collected light is filtered by a 568-nm long-pass filter and a 532-nm notch filter. The excitation and collection spots are aligned to the same spot, over the center of the cavity.

For the $g^{(2)}$ measurement, the excitation power is set to $450 \mu \mathrm{W}$. The light collected by the single-mode fiber is filtered by a monochromator (Princeton Instruments Acton SP2750). The light is then sent via multimode fiber to a Hanbury Brown and Twiss setup comprising a fiber beam splitter and two single-photon counting modules (SPCM) (PerkinElmer SPCM-AQR-14-FC). A time-correlated single-photon counting module (PicoHarp 300) was used to construct the histogram shown in Fig. 2(d).
To measure the lifetimes of the $\mathrm{SnV}^{-}$center in the cavity presented in Fig. 3, we used a supercontinuum laser (Fianium SC400) filtered with a 450-nm-long-pass filter and a 550-nm short-pass filter to excite the $\mathrm{SnV}^{-}$center. The emitted light was then filtered by a 568-nm long-pass filter before being coupled into a fiber. The collected light was further filtered by a monochromator (Princeton Instruments Acton SP2750) before being coupled into a multimode fiber and sent to a SPCM. The lifetime measurements were recorded using the time-correlated single-photon counting module.

[1] H. J. Kimble, The Quantum Internet, Nature (London) 453, 1023 (2008).

[2] A. Reiserer and G. Rempe, Cavity-Based Quantum Networks with Single Atoms and Optical Photons, Rev. Mod. Phys. 87, 1379 (2015).

[3] C. Monroe and J. Kim, Scaling the Ion Trap Quantum Processor, Science 339, 1164 (2013).

[4] D. D. Awschalom, R. Hanson, J. Wrachtrup, and B. B. Zhou, Quantum Technologies with Optically Interfaced Solid-State Spins, Nat. Photonics 12, 516 (2018).

[5] M. Atatüre, D. Englund, N. Vamivakas, S.-Y. Lee, and J. Wrachtrup, Material Platforms for Spin-Based Photonic Quantum Technologies, Nat. Rev. Mater. 3, 38 (2018).

[6] E. Janitz, M. K. Bhaskar, and L. Childress, Cavity Quantum Electrodynamics with Color Centers in Diamond, Optica 7, 1232 (2020).

[7] B. Hensen, H. Bernien, A. E. Dréau, A. Reiserer, N. Kalb, M. S. Blok, J. Ruitenberg, R. F. L. Vermeulen, R. N. Schouten, C. Abellán, W. Amaya, V. Pruneri, M. W. Mitchell, M. Markham, D. J. Twitchen, D. Elkouss, S. Wehner, T. H. Taminiau, and R. Hanson, Loophole-Free Bell Inequality Violation Using Electron Spins Separated by 1.3 Kilometres, Nature (London) 526, 682 (2015).

[8] N. Kalb, A. A. Reiserer, P. C. Humphreys, J. J. W. Bakermans, S. J. Kamerling, N. H. Nickerson, S. C. Benjamin, D. J. Twitchen, M. Markham, and R. Hanson, Entanglement Distillation between Solid-State Quantum Network Nodes, Science 356, 928 (2017).

[9] A. Faraon, C. Santori, Z. Huang, V. M. Acosta, and R. G. Beausoleil, Coupling of Nitrogen-Vacancy Centers to Photonic Crystal Cavities in Monocrystalline Diamond, Phys. Rev. Lett. 109, 033604 (2012).

[10] L. Li, T. Schröder, E. H. Chen, M. Walsh, I. Bayn, J. Goldstein, O. Gaathon, M. E. Trusheim, M. Lu, J. Mower, M. Cotlet, M. L. Markham, D. J. Twitchen, and D. Englund, Coherent Spin Control of a Nanocavity-Enhanced Qubit in Diamond, Nat. Commun. 6, 6173 (2015).

[11] C. Bradac, W. Gao, J. Forneris, M. E. Trusheim, and I. Aharonovich, Quantum Nanophotonics with Group IV Defects in Diamond, Nat. Commun. 10, 5625 (2019).

[12] N. H. Wan, T.-J. Lu, K. C. Chen, M. P. Walsh, M.E. Trusheim, L. De Santis, E. A. Bersin, I. B. Harris, S. L. Mouradian, I. R. Christen, E. S. Bielejec, and D. Englund, Large-Scale Integration of Artificial Atoms in Hybrid Photonic Circuits, Nature (London) 583, 226 (2020). 
[13] M. E. Trusheim, B. Pingault, N. H. Wan, M. Gündoğan, L. De Santis, R. Debroux, D. Gangloff, C. Purser, K. C. Chen, M. Walsh et al., Transform-Limited Photons from a Coherent Tin-Vacancy Spin in Diamond, Phys. Rev. Lett. 124, 023602 (2020).

[14] A. E. Rugar, C. Dory, S. Aghaeimeibodi, H. Lu, S. Sun, S. D. Mishra, Z.-X. Shen, N. A. Melosh, and J. Vučković, Narrow-Linewidth Tin-Vacancy Centers in a Diamond Waveguide, ACS Photonics 7, 2356 (2020).

[15] M. K. Bhaskar, R. Riedinger, B. Machielse, D. S. Levonian, C. T. Nguyen, E. N. Knall, H. Park, D. Englund, M. Lončar, D. D. Sukachev, and M. D. Lukin, Experimental Demonstration of Memory-Enhanced Quantum Communication, Nature (London) 580, 60 (2020).

[16] Y.-I. Sohn, S. Meesala, B. Pingault, H. A. Atikian, J. Holzgrafe, M. Gündoğan, C. Stavrakas, M. J. Stanley, A. Sipahigil, J. Choi, M. Zhang, J. L. Pacheco, J. Abraham, E. Bielejec, M. D. Lukin, M. Atatüre, and M. Lončar, Controlling the Coherence of a Diamond Spin Qubit through Its Strain Environment, Nat. Commun. 9, 2012 (2018).

[17] D. D. Sukachev, A. Sipahigil, C. T. Nguyen, M. K. Bhaskar, R. E. Evans, F. Jelezko, and M. D. Lukin, Silicon-Vacancy Spin Qubit in Diamond: A Quantum Memory Exceeding 10 ms with Single-Shot State Readout, Phys. Rev. Lett. 119, 223602 (2017).

[18] G. Thiering and A. Gali, Ab Initio Magneto-Optical Spectrum of Group-IV Vacancy Color Centers in Diamond, Phys. Rev. X 8, 021063 (2018).

[19] T. Iwasaki, Y. Miyamoto, T. Taniguchi, P. Siyushev, M. H. Metsch, F. Jelezko, and M. Hatano, Tin-Vacancy Quantum Emitters in Diamond, Phys. Rev. Lett. 119, 253601 (2017).

[20] A. E. Rugar, C. Dory, S. Sun, and J. Vučković, Characterization of Optical and Spin Properties of Single TinVacancy Centers in Diamond Nanopillars, Phys. Rev. B 99, 205417 (2019).

[21] J. Görlitz, D. Herrmann, G. Thiering, P. Fuchs, M. Gandil, T. Iwasaki, T. Taniguchi, M. Kieschnick, J. Meijer, M. Hatano, A. Gali, and C. Becher, Spectroscopic Investigations of Negatively Charged Tin-Vacancy Centres in Diamond, New J. Phys. 22, 013048 (2020).

[22] B. Khanaliloo, M. Mitchell, A. C. Hryciw, and P. E. Barclay, High-Q/V Monolithic Diamond Microdisks Fabricated with Quasi-isotropic Etching, Nano Lett. 15, 5131 (2015).

[23] S. Mouradian, N. H. Wan, T. Schröder, and D. Englund, Rectangular Photonic Crystal Nanobeam Cavities in Bulk Diamond, Appl. Phys. Lett. 111, 021103 (2017).

[24] N. H. Wan, S. Mouradian, and D. Englund, Two-Dimensional Photonic Crystal Slab Nanocavities on Bulk Single-Crystal Diamond, Appl. Phys. Lett. 112, 141102 (2018).

[25] M. Mitchell, D. P. Lake, and P.E. Barclay, Realizing $Q>300000$ in Diamond Microdisks for Optomechanics via Etch Optimization, APL Photonics 4, 016101 (2019).
[26] C. Dory, D. Vercruysse, K. Y. Yang, N. V. Sapra, A. E. Rugar, S. Sun, D. M. Lukin, A. Y. Piggott, J. L. Zhang, M. Radulaski, K. G. Lagoudakis, L. Su, and J. Vučković, Inverse-Designed Diamond Photonics, Nat. Commun. 10, 3309 (2019).

[27] Y. Gong, B. Ellis, G. Shambat, T. Sarmiento, J. S. Harris, and J. Vuckovic, Nanobeam Photonic Crystal Cavity Quantum Dot Laser, Opt. Express 18, 8781 (2010).

[28] A. E. Rugar, H. Lu, C. Dory, S. Sun, P. J. McQuade, Z.-X. Shen, N. A. Melosh, and J. Vučković, Generation of TinVacancy Centers in Diamond via Shallow Ion Implantation and Subsequent Diamond Overgrowth, Nano Lett. 20, 1614 (2020).

[29] D. Riedel, I. Söllner, B. J. Shields, S. Starosielec, P. Appel, E. Neu, P. Maletinsky, and R. J. Warburton, Deterministic Enhancement of Coherent Photon Generation from a Nitrogen-Vacancy Center in Ultrapure Diamond, Phys. Rev. X 7, 031040 (2017).

[30] D. M. Toyli, C. D. Weis, G. D. Fuchs, T. Schenkel, and D. D. Awschalom, Chip-Scale Nanofabrication of Single Spins and Spin Arrays in Diamond, Nano Lett. 10, 3168 (2010).

[31] I. Bayn, E. H. Chen, M. E. Trusheim, L. Li, T. Schröder, O. Gaathon, M. Lu, A. Stein, M. Liu, K. Kisslinger, H. Clevenson, and D. Englund, Generation of Ensembles of Individually Resolvable Nitrogen Vacancies Using Nanometer-Scale Apertures in Ultrahigh-Aspect Ratio Planar Implantation Masks, Nano Lett. 15, 1751 (2015).

[32] D. Scarabelli, M. Trusheim, O. Gaathon, D. Englund, and S. J. Wind, Nanoscale Engineering of Closely-Spaced Electronic Spins in Diamond, Nano Lett. 16, 4982 (2016).

[33] S. Wehner, D. Elkouss, and R. Hanson, Quantum Internet: A Vision for the Road Ahead, Science 362, eaam9288 (2018).

[34] M. J. Burek, C. Meuwly, R. E. Evans, M. K. Bhaskar, A. Sipahigil, S. Meesala, B. Machielse, D. D. Sukachev, C. T. Nguyen, J. L. Pacheco, E. Bielejec, M. D. Lukin, and M. Lončar, Fiber-Coupled Diamond Quantum Nanophotonic Interface, Phys. Rev. Applied 8, 024026 (2017).

[35] A. W. Elshaari, W. Pernice, K. Srinivasan, O. Benson, and V. Zwiller, Hybrid Integrated Quantum Photonic Circuits, Nat. Photonics 14, 285 (2020).

[36] J. Kim, S. Aghaeimeibodi, J. Carolan, D. Englund, and E. Waks, Hybrid Integration Methods for On-Chip Quantum Photonics, Optica 7, 291 (2020).

[37] K. Kuruma, B. Pingault, C. Chia, D. Renaud, P. Hoffmann, S. Iwamoto, C. Ronning, and M. Lončar, Coupling of a Single Tin-Vacancy Center to a Photonic Crystal Cavity in Diamond, arXiv:2105.01715. 